AperTO - Archivio Istituzionale Open Access dell'Università di Torino

\title{
Differentiation Based on Optimal Local Spline Quasi-Interpolants with Applications
}

\section{This is the author's manuscript}

Original Citation:

\section{Availability:}

This version is available http://hdl.handle.net/2318/75931

since

Publisher:

American Institute of Physics (AIP)

Published version:

DOI:10.1063/1.3498341

Terms of use:

Open Access

Anyone can freely access the full text of works made available as "Open Access". Works made available under a Creative Commons license can be used according to the terms and conditions of said license. Use of all other works requires consent of the right holder (author or publisher) if not exempted from copyright protection by the applicable law. 


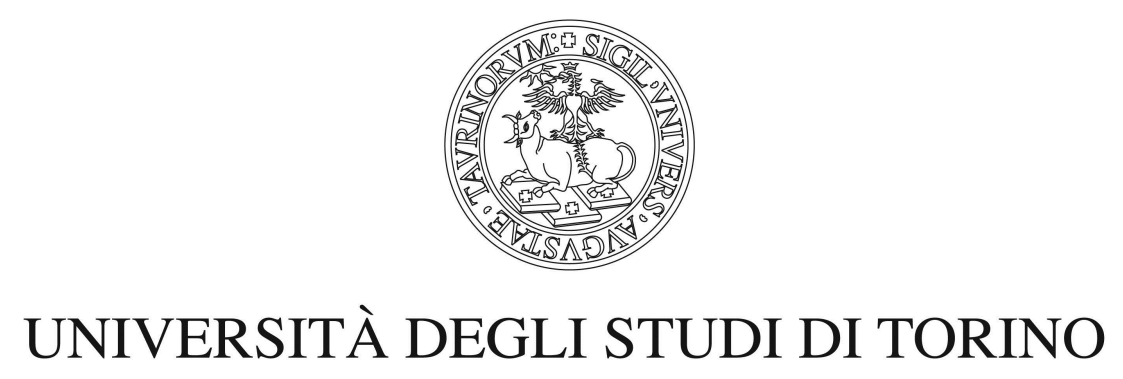

This is an author version of the contribution published on:

Catterina Dagnino and Sara Remogna. Differentiation Based on Optimal Local Spline Quasi-Interpolants with Applications. AIP Conference Proceedings, 1281, 2010, DOI 10.1063/1.3498341.

The definitive version is available at:

http://scitation.aip.org/content/aip/proceeding/aipcp/10.1063/1.3498341 


\title{
Differentiation Based on Optimal Local Spline Quasi-Interpolants with Applications
}

\author{
Catterina Dagnino and Sara Remogna \\ Department of Mathematics, University of Torino, via C. Alberto, 10 - 10123 Torino, Italy
}

\begin{abstract}
In this paper we propose a method for the approximation of the derivative of a function $f$ based on discrete local optimal spline quasi-interpolants $Q_{k}$ of degree $k=3,4,5$. By differentiating $Q_{k} f$, we construct the approximation of the derivative at the quasi-interpolation nodes and the corresponding differentiation matrices. Some numerical results and applications to univariate boundary-value problems are given.
\end{abstract}

Keywords: Spline quasi-interpolants, Numerical differentiation, Spline collocation methods

PACS: 02.60.-x, 02.60.Jh, 02.70.Jn

\section{INTRODUCTION}

Local spline quasi-interpolation has been widely analysed in approximation theory (see e.g. $[1,3,5,7]$ and references therein), but only recently some studies have been devoted to its applications in the numerical differentiation $[2,4,6]$. In particular, in [2], the authors focus on approximations of first and second derivatives by those of local quadratic spline quasi-interpolants and their applications to collocation methods. The proposed formulas are very accurate at some points, thanks to the superconvergence properties of those operators and they give rise to good global approximations of derivatives on the whole domain of definition. In this paper, we propose differentiation formulas still based on discrete local spline quasi-interpolants, but of higher degree. We get differentiation matrices, that we use in collocation methods for the solution of some univariate boundary-value problems. We propose the same numerical tests of [2] and we show that spline quasi-interpolants of higher degree and smoothness provide very accurate results. Indeed, the results obtained with cubic splines are comparable to those given in [2] for quadratic case, as we expect from the theory, instead we obtain higher performances with quartic and quintic splines.

\section{APPROXIMATION OF FIRST DERIVATIVES AND DIFFERENTIATION MATRICES}

Let $I=[a, b]$ be a bounded interval endowed with a uniform knot partition $\Delta_{n}=\left\{x_{i}=a+i h, 0 \leq i \leq n\right\}$, with $h=(b-a) / n$ and $k$ a non-negative integer. We define the spline space $\mathscr{S}_{k}^{k-1}\left(\Delta_{n}\right)=\left\{s \in C^{k-1}(I):\left.s\right|_{\left[x_{i}, x_{i+1}\right)} \in \mathbb{P}_{k}, i=\right.$ $0,1, \ldots, n-1\}$ where $\mathbb{P}_{k}$ denotes the space of polynomials in $x$ of degree $k$.

We denote by $\left\{N_{j}^{k}(x)\right\}_{j=1}^{n+k}$ the basis of normalized B-splines defined on the extended knot partition $\left\{x_{-k}=\ldots=\right.$ $\left.x_{-1}=x_{0}=a, x_{i}, \quad 1 \leq i \leq n-1, b=x_{n}=x_{n+1}=\ldots=x_{n+k}\right\}$ and spanning $\mathscr{S}_{k}^{k-1}\left(\Delta_{n}\right)$ [1]. With our notations the support of the B-spline $N_{j}^{k}$ is $\left[x_{j-k-1}, x_{j}\right]$.

We define the following two sets of quasi-interpolation nodes:

- $\mathscr{T}_{n}^{1}=\left\{t_{i}, i=1, \ldots, n+2\right\}$, where $t_{1}=a, t_{i}=\frac{1}{2}\left(x_{i-2}+x_{i-1}\right)$, for $i=2, \ldots, n+1, t_{n+2}=b$, in case $k$ even;

- $\mathscr{T}_{n}^{2}=\left\{t_{i}, i=1, \ldots, n+1\right\}$, where $t_{1}=a, t_{i}=x_{i-1}$, for $i=2, \ldots, n, t_{n+1}=b$, in case $k$ odd.

Let $f$ be a smooth function, we set $f_{j}=f\left(t_{j}\right)$ and we consider the spline quasi-interpolants (QI)

$$
Q_{k} f(x)=\sum_{j=1}^{n+k} m_{j}(f) N_{j}^{k}(x)= \begin{cases}\sum_{j=1}^{n+2} f_{j} \widetilde{N}_{j}^{k}(x) & k \text { even } \\ \sum_{j=1}^{n+1} f_{j} \widetilde{N}_{j}^{k}(x) & k \text { odd }\end{cases}
$$

with

- $m_{j}(f)=\sum_{i} \lambda_{i, j} f\left(t_{i}\right), \lambda_{i, j} \in \mathbb{R}$, local linear functionals defined as combinations of discrete values of $f$ at the points $t_{i}$ lying in the support (or near the support) of $N_{j}^{k}$, given in [6] for $k=2, \ldots, 5$ and constructed so that $Q_{k}$ is exact 
on $\mathbb{P}_{k}$, i.e. $Q_{k} p=p, p \in \mathbb{P}_{k}$. Consequently, $\left\|f-Q_{k} f\right\|_{\infty}=O\left(h^{k+1}\right)$, for $f \in C^{k+1}(I)$;

- $\widetilde{N}_{j}^{k}$ the so-called fundamental functions associated with $Q_{k}$, given by linear combinations of B-splines $N_{j}^{k}$ and obtained, after some algebra, from the expression of the functionals $m_{j}$.

We approximate the derivative of $f$ by the derivative of $Q_{k} f$. We denote it by $Q_{k}^{\prime} f$ and we remark that it belongs to $\mathscr{S}_{k-1}^{k-2}\left(\Delta_{n}\right)$, the spline space of degree $k-1$ defined on $\Delta_{n}$. From (1), we obtain

$$
Q_{k}^{\prime} f(x)=\left(\sum_{j=1}^{n+k} m_{j}(f) N_{j}^{k}(x)\right)^{\prime}= \begin{cases}\sum_{j=1}^{n+2} f_{j}\left(\widetilde{N}_{j}^{k}\right)^{\prime}(x) & k \text { even } \\ \sum_{j=1}^{n+1} f_{j}\left(\widetilde{N}_{j}^{k}\right)^{\prime}(x) & k \text { odd. }\end{cases}
$$

If we approximate $f^{\prime}$ at the quasi-interpolation nodes we have to evaluate (2) at the points of $\mathscr{T}_{n}^{1}$ if $k$ is even and $\mathscr{T}_{n}^{2}$ if $k$ is odd. The values $\left(\widetilde{N}_{j}^{k}\right)^{\prime}\left(t_{i}\right)$, computed using the differentiation formula for the B-splines $N_{j}^{k}$ (see [1]), can be stored in the differentiation matrix $D_{k}$, where $D_{k} \in \mathbb{R}^{(n+2) \times(n+2)}$ for $k$ even and $D_{k} \in \mathbb{R}^{(n+1) \times(n+1)}$ for $k$ odd.

Setting $\mathbf{y}$ for the vector with components $y_{j}=f\left(t_{j}\right)$ and $\mathbf{y}^{\prime}$ for the vector with components $y_{j}^{\prime}=Q_{k}^{\prime} f\left(t_{j}\right)$, we get:

$$
\mathbf{y}^{\prime}=D_{k} \mathbf{y}
$$

where the matrix $D_{k}$ has elements $d_{i j}=\left(\widetilde{N}_{j}^{k}\right)^{\prime}\left(t_{i}\right)$ and the following structure:

$D_{k}=\frac{1}{h}\left(\begin{array}{c}D_{k}^{(1)} \\ D_{k}^{(2)} \\ D_{k}^{(3)}\end{array}\right), \quad$ with $\quad D_{k}^{(1)}, D_{k}^{(3)} \in\left\{\begin{array}{l}\mathbb{R}^{(k+1) \times(n+2)} \text { if } k \text { even } \\ \mathbb{R}^{(k-1) \times(n+1)} \text { if } k \text { odd }\end{array} \quad, \quad D_{k}^{(2)} \in\left\{\begin{array}{l}\mathbb{R}^{(n-2 k) \times(n+2)} \text { if } k \text { even } \\ \mathbb{R}^{(n-2 k+3) \times(n+1)} \text { if } k \text { odd }\end{array}\right.\right.$ and

$$
\begin{aligned}
& \cdot D_{k}^{(1)}=\left(d_{i j}\right)\left\{\begin{array}{lll}
\neq 0 & 1 \leq i \leq k+1,1 \leq j \leq i+k, & \text { if } k \text { even } \\
\neq 0 & i=1,1 \leq j \leq i+k ; 2 \leq i \leq k-1,1 \leq j \leq i+k-1, & \text { if } k \text { odd } \\
=0 & \text { otherwise }
\end{array}\right. \\
& \cdot D_{k}^{(2)}=\left(d_{i j}\right)\left\{\begin{array}{lll}
\neq 0 & k+2 \leq i \leq n-k+1, i-k \leq j \leq i+k, j \neq i & \text { if } k \text { even } \\
\neq 0 & k \leq i \leq n-k+2, i-k+1 \leq j \leq i+k-1, j \neq i & \text { if } k \text { odd } \\
=0 & \text { otherwise }
\end{array}\right. \\
& \cdot D_{k}^{(3)}=\left(d_{i j}\right)\left\{\begin{array}{lll}
=-d_{n-i+3, n-j+3} & n-k+2 \leq i \leq n+2, i-k \leq j \leq n+2, \\
=-d_{n-i+2, n-j+2} & n-k+3 \leq i \leq n, i-k+1 \leq j \leq n+1 ; i=n+1, i-k \leq j \leq n+1, & \text { if } k \text { oden } \\
=0 & \text { otherwise. }
\end{array}\right.
\end{aligned}
$$

Now we report the nonzero elements of the differentiation matrices for quartic and quintic splines, i.e. $k=4$ and $k=5$, respectively:

- $D_{4}$

$-D_{4}^{(1)} \in \mathbb{R}^{5 \times(n+2)}$ :

$\begin{array}{llllrl}d_{11}=-352 / 105, & d_{12}=35 / 8, & d_{13}=-35 / 24, & d_{14}=21 / 40, & d_{15}=-5 / 56, \\ d_{21}=-13871 / 15120, & d_{22}=357 / 2048, & d_{23}=4561 / 4608, & d_{24}=-1489 / 5120, & & d_{25}=155 / 3584, \\ d_{26}=47 / 55296, & & & & & \\ d_{31}=3767 / 15120, & d_{32}=-23887 / 27648, & d_{33}=-641 / 55296, & d_{34}=26119 / 34560, & & d_{35}=-27317 / 193536, \\ d_{36}=101 / 9216, & d_{37}=47 / 55296, & & & \\ d_{41}=-841 / 15120, & d_{42}=1417 / 6912, & d_{43}=-7133 / 9216, & d_{44}=1513 / 92160, & d_{45}=35419 / 48384, \\ d_{46}=-3751 / 27648, & d_{47}=101 / 9216, & d_{48}=47 / 55296, & & \\ d_{51}=-47 / 15120, & d_{52}=-209 / 27648, & d_{53}=463 / 3456, & d_{54}=-101521 / 138240, & d_{55}=-47 / 387072, \\ d_{56}=20323 / 27648, & d_{57}=-3751 / 27648, & d_{58}=101 / 9216, & d_{59}=47 / 55296, & \end{array}$

$-D_{4}^{(2)} \in \mathbb{R}^{(n-8) \times(n+2)}$ :

$d_{i, i-4}=-d_{i, i+4}=-47 / 55296, \quad d_{i, i-3}=-d_{i, i+3}=-101 / 9216, \quad d_{i, i-2}=-d_{i, i+2}=3751 / 27648$,

$d_{i, i-1}=-d_{i, i+1}=-20323 / 27648, \quad d_{i, i}=0, \quad i=6, \ldots, n-3$,

- $D_{4}^{(3)} \in \mathbb{R}^{5 \times(n+2)}$, defined by the elements of $D_{4}^{(1)}$. 
- $D_{5}$

$$
\begin{aligned}
& \text { - } D_{5}^{(1)} \in \mathbb{R}^{4 \times(n+1)} \text { : } \\
& d_{11}=-137 / 60, \quad d_{12}=5, \quad d_{13}=-5, \quad d_{14}=10 / 3, \quad d_{15}=-5 / 4, \quad d_{16}=1 / 5, \\
& d_{21}=-1 / 5, \quad d_{22}=-13 / 12, \quad d_{23}=2, \quad d_{24}=-1, \quad d_{25}=1 / 3, \quad d_{26}=-1 / 20, \\
& d_{31}=301 / 5760, \quad d_{32}=-493 / 960, \quad d_{33}=-115 / 384, \quad d_{34}=275 / 288, \quad d_{35}=-83 / 384, \quad d_{36}=19 / 960, \\
& \begin{array}{l}
d_{37}=13 / 5760, \quad d_{42}=877 / 5760, \quad d_{43}=-733 / 960, \quad d_{44}=13 / 384, \quad d_{45}=203 / 288, \quad d_{46}=-223 / 1920, \\
d_{41}=-1 / 60, \quad, \quad d_{40}=13 / 5760,
\end{array} \\
& d_{47}=1 / 320, \quad d_{48}=13 / 5760 \\
& -D_{5}^{(2)} \in \mathbb{R}^{(n-7) \times(n+1)} \text { : } \\
& d_{i, i-4}=-d_{i, i+4}=-13 / 5760, \quad d_{i, i-3}=-d_{i, i+3}=-1 / 320, \quad d_{i, i-2}=-d_{i, i+2}=341 / 2880, \\
& d_{i, i-1}=-d_{i, i+1}=-2069 / 2880, \quad d_{i, i}=0, \quad i=5, \ldots, n-3, \\
& \text { - } D_{5}^{(3)} \in \mathbb{R}^{4 \times(n+1)} \text {, defined by the elements of } D_{5}^{(1)} \text {. }
\end{aligned}
$$

For the cubic case $k=3$ see [6], where the matrix $D_{3}$ is given.

In order to analyse the error $\left(f-Q_{k} f\right)^{\prime}$ at the quasi-interpolation nodes $t_{i}$, we consider (3) and we compute the Taylor expansion of $f$ at these nodes. With the help of a computer algebra system, we get the following results.

Proposition 1 For sufficiently smooth functions $f$, the error at the quasi-interpolation nodes $t_{i}$ is given by:

- for $k=3$

$$
\begin{aligned}
& \left|y_{i}^{\prime}-f_{i}^{\prime}\right|=\frac{h^{3}}{4}\left|f_{i}^{(4)}\right|+O\left(h^{4}\right), i=1, n+1, \quad\left|y_{i}^{\prime}-f_{i}^{\prime}\right|=\frac{h^{3}}{12}\left|f_{i}^{(4)}\right|+O\left(h^{4}\right), i=2, n, \\
& \left|y_{i}^{\prime}-f_{i}^{\prime}\right|=\frac{h^{4}}{30}\left|f_{i}^{(5)}\right|+O\left(h^{6}\right), i=3, \ldots, n-1
\end{aligned}
$$

- for $k=4$

$$
\begin{aligned}
& \left|y_{i}^{\prime}-f_{i}^{\prime}\right|=\frac{7}{128} h^{4}\left|f_{i}^{(5)}\right|+O\left(h^{5}\right), i=1, n+2, \quad\left|y_{i}^{\prime}-f_{i}^{\prime}\right|=\frac{1583}{61440} h^{4}\left|f_{i}^{(5)}\right|+O\left(h^{5}\right), i=2, n+1, \\
& \left|y_{i}^{\prime}-f_{i}^{\prime}\right|=\frac{1157}{110592} h^{4}\left|f_{i}^{(5)}\right|+O\left(h^{5}\right), i=3, n, \quad\left|y_{i}^{\prime}-f_{i}^{\prime}\right|=\frac{661}{184320} h^{4}\left|f_{i}^{(5)}\right|+O\left(h^{5}\right), i=4, n-1, \\
& \left|y_{i}^{\prime}-f_{i}^{\prime}\right|=\frac{719}{552960} h^{4}\left|f_{i}^{(5)}\right|+O\left(h^{5}\right), i=5, n-2, \quad\left|y_{i}^{\prime}-f_{i}^{\prime}\right|=\frac{7}{5760} h^{4}\left|f_{i}^{(5)}\right|+O\left(h^{6}\right), i=6, \ldots, n-3 .
\end{aligned}
$$

- for $k=5$

$$
\begin{aligned}
& \left|y_{i}^{\prime}-f_{i}^{\prime}\right|=\frac{h^{5}}{6}\left|f_{i}^{(6)}\right|+O\left(h^{6}\right), i=1, n+1, \quad\left|y_{i}^{\prime}-f_{i}^{\prime}\right|=\frac{h^{5}}{30}\left|f_{i}^{(6)}\right|+O\left(h^{6}\right), i=2, n, \\
& \left|y_{i}^{\prime}-f_{i}^{\prime}\right|=\frac{109}{5760} h^{5}\left|f_{i}^{(6)}\right|+O\left(h^{6}\right), i=3, n-1, \quad\left|y_{i}^{\prime}-f_{i}^{\prime}\right|=\frac{13}{5760} h^{5}\left|f_{i}^{(6)}\right|+O\left(h^{6}\right), i=4, n-2, \\
& \left|y_{i}^{\prime}-f_{i}^{\prime}\right|=\frac{47}{4032} h^{6}\left|f_{i}^{(7)}\right|+O\left(h^{8}\right), \quad i=5, \ldots, n-3 .
\end{aligned}
$$

Since the global approximation error $\left(f-Q_{k} f\right)^{\prime}$ is $O\left(h^{k}\right)$ [3], there appears a superconvergence phenomenon for the odd cases $k=3,5$ at the inner quasi-interpolation nodes.

Now we propose the following two examples of approximation of derivatives by the above formulas, in case $k=3,4,5$ and we compare them with the results obtained in [2] by differentiation formulas based on quadratic quasi-interpolating splines. The test functions are $f=\phi_{j}, j=1,2$ on $I=[-1,1]$, with $\phi_{1}(x)=\frac{1}{4}\left(1-x^{2}\right)^{2}$ and $\phi_{2}(x)=\sin (\pi x)+\sin (5 \pi x)$. We compute $\max _{v \in V}\left|f^{\prime}(v)-Q_{k}^{\prime} f(v)\right|$, where $V=\mathscr{T}_{n}^{1}$ for $k$ even and $V=\mathscr{T}_{n}^{2}$ for $k$ odd. These maximum absolute errors are reported in Table 1 for increasing values of $n$ and they confirm the results of Proposition 1 . As expected, we can notice that $\phi_{1}^{\prime}$ is exactly reproduced using quartic and quintic splines.

\section{COLLOCATION METHODS FOR UNIVARIATE BOUNDARY-VALUE PROBLEMS}

We consider the following boundary-value problem

$$
\left\{\begin{array}{l}
\frac{d}{d x}\left(p(x) \frac{d u(x)}{d x}\right)+r(x) u(x)=f(x), \quad \text { for } x \in I \\
u(a)=u(b)=0
\end{array}\right.
$$


TABLE 1. Maximum absolute errors.

\begin{tabular}{|c|c|c|c|c|c|c|c|c|c|c|}
\hline$n$ & & $k=2[2]$ & $k=3$ & $k=4$ & $k=5$ & & $k=2$ [2] & $k=3$ & $k=4$ & $k=5$ \\
\hline 8 & & $6.5(-3)$ & $2.3(-2)$ & $5.6(-17)$ & $5.5(-17)$ & & 30.8 & 30.9 & 19.7 & 58.9 \\
\hline 16 & & $8.1(-4)$ & $2.9(-3)$ & $1.4(-16)$ & $1.4(-16)$ & & 13.9 & 14.0 & 6.6 & 28.7 \\
\hline 32 & $\phi_{1}$ & $1.0(-4)$ & $3.7(-4)$ & $3.1(-16)$ & $1.7(-16)$ & $\phi_{2}$ & 3.1 & 3.1 & $1.9(-1)$ & 1.7 \\
\hline 64 & & $1.3(-5)$ & $4.6(-5)$ & $7.8(-16)$ & $6.7(-16)$ & & $2.5(-1)$ & $2.5(-1)$ & $3.8(-2)$ & $6.2(-2)$ \\
\hline 128 & & $1.6(-6)$ & $5.7(-6)$ & $1.6(-15)$ & $1.8(-15)$ & & $1.7(-2)$ & $1.7(-2)$ & $2.9(-3)$ & $1.2(-3)$ \\
\hline
\end{tabular}

where $p \in C^{1}(I), r \in C(I)$ and $f$ is a piecewise continuous function on $I$. We can use the QIs previously considered and their differentiation matrices to solve this problem.

Let $\mathbf{u}$ be the vector of unknown values of $u$ at the points $\left\{t_{i}, i \in \mathscr{I}\right\}, \mathscr{I}=\{2, \ldots, n+1\}$ if $k$ is even and $\mathscr{I}=\{2, \ldots, n\}$ if $k$ is odd, and let $\tilde{\mathbf{u}}$ be the augmented vector with 0 as first and last elements. Considering $D_{k} \tilde{\mathbf{u}}$ we obtain an approximation of $u^{\prime}$ at the quasi-interpolation nodes. Then, we multiply this vector by the diagonal matrix $P$, with $P(i, i)=p\left(t_{i}\right)\left(t_{i} \in \mathscr{T}_{n}^{1}\right.$ if $k$ is even, $t_{i} \in \mathscr{T}_{n}^{2}$ if $k$ is odd) and we multiply again by $D_{k}$. Denoting by $R$ the diagonal matrix defined by $R(i, i)=r\left(t_{i}\right)\left(t_{i} \in \mathscr{T}_{n}^{1}\right.$ if $k$ is even, $t_{i} \in \mathscr{T}_{n}^{2}$ if $k$ is odd), we see that the left-hand side of the differential equation (4) is approximated by the vector

$$
\tilde{A} \tilde{\mathbf{u}}:=\left(-D_{k} P D_{k}+R\right) \tilde{\mathbf{u}} .
$$

Let $A$ be the matrix deduced from $\tilde{A}$ by deleting the first and last rows and columns, it is well-known ([8] Chap.7) that the problem is equivalent to solve the linear system $A \mathbf{u}=\mathbf{f}$, where $\mathbf{f}$ is the vector of components $f\left(t_{i}\right), i \in \mathscr{I}$.

We propose the following two test problems:

Test 1: $\quad-u^{\prime \prime}(x)+u(x)=\left(1+\pi^{2}\right) \sin (\pi x), \quad u(-1)=u(1)=0$, where $u(x)=\sin (\pi x)$.

Test 2: $\quad-u^{\prime \prime}(x)+u(x)=\exp \left(\frac{x}{2}\right)\left(\left(100 \pi^{2}+\frac{3}{4}\right) \sin (10 \pi x)-10 \pi \cos (10 \pi x)\right)+\left(9 \pi^{2}+1\right) \sin (3 \pi x)$,

$$
u(-1)=u(1)=0 \text {, where } u(x)=\exp \left(\frac{x}{2}\right) \sin (10 \pi x)+\sin (3 \pi x) \text {. }
$$

We compute $\max _{i \in \mathscr{I}}\left|u\left(t_{i}\right)-\mathbf{u}(i)\right|$, reporting our results in Table 2 , for $k=3,4,5$. If we compare them with those obtained in [2], in case $k=2$ (also reported in Table 2), we can remark the good performances of the formulas based on high degree QIs.

\begin{tabular}{|c|c|c|c|c|c|c|c|c|c|c|}
\hline$n$ & & $k=2[2]$ & $k=3$ & $k=4$ & $k=5$ & & $k=2[2]$ & $k=3$ & $k=4$ & $k=5$ \\
\hline 8 & & $1.8(-2)$ & $2.3(-2)$ & $5.4(-3)$ & $3.6(-3)$ & & 39 & 41 & 34 & 38 \\
\hline 16 & & $1.2(-3)$ & $1.3(-3)$ & $2.1(-4)$ & $1.0(-4)$ & & 22 & 22 & 16 & 19 \\
\hline 32 & Test 1 & $7.6(-5)$ & $8.2(-5)$ & 7.6(-6) & $1.6(-6)$ & Test 2 & 2.9 & 3.4 & 1.5 & 2.8 \\
\hline 64 & & $4.8(-6)$ & $5.3(-6)$ & $3.3(-7)$ & $2.2(-8)$ & & $1.2(-1)$ & $2.4(-1)$ & $2.3(-2)$ & $5.3(-2)$ \\
\hline 128 & & $3.0(-7)$ & $3.4(-7)$ & $1.7(-8)$ & $3.2(-10)$ & & $6.3(-3)$ & $1.4(-2)$ & $1.2(-3)$ & $9.7(-4)$ \\
\hline
\end{tabular}

TABLE 2. Maximum absolute errors.

\section{REFERENCES}

1. C. de Boor, A practical guide to splines (Revised Edition), Springer-Verlag, New York, 2001.

2. F. Foucher and P. Sablonnière, "Quadratic spline quasi-interpolants and collocation methods," Math. Comput. Simulation 79, 3455-3465 (2009).

3. T. Lyche and L. L. Schumaker, "Local spline approximation methods," J. Approx. Theory 15, 294-325 (1975).

4. S. Remogna, "Pseudo-spectral derivative of quadratic quasi-interpolant splines," Rend. Sem. Mat. Univ. Pol. Torino 67, 351-362 (2009).

5. P. Sablonnière, "Quadratic spline quasi-interpolants on bounded domains of $\mathbb{R}^{d}, d=1,2,3$, , Rend. Sem. Mat. Univ. Pol. Torino 61, 229-246 (2003).

6. P. Sablonnière, "Univariate spline quasi-interpolants and applications to numerical analysis," Rend. Sem. Mat. Univ. Pol. Torino 63, 211-222 (2005)

7. P. Sablonnière, "Recent progress on univariate and multivariate polynomial and spline quasi-interpolants," in Trends and Applications in Constructive Approximation, edited by M. G. De Bruijn et al., Internat. Ser. Numer. Math. 151, Birkhäuser, Basel, 2005, pp. 229-245.

8. L. N. Trefethen, Spectral Methods in Matlab, SIAM, Philadelphia, 2000. 